\title{
Steckt die Verhaltenstherapie bei Kindern noch in den Kinderschuhen?
}

Die Verhaltenstherapie hat ihren Ursprung nicht zuletzt in der Behandlung psychischer Störungen des Kindesalters. So berichtete bereits 1924 Mary Cover Jones über die systematische Anwendung verhaltenstherapeutischer Techniken bei der Behandlung der Pelztierphobie des kleinen Peters. Mowrer und Mowrer publizierten 1938 den verhaltenstherapeutischen Einsatz der Klingelmatte zur Behandlung der funktionellen Enuresis. Angesichts dieser Initialwirkung verhaltenstherapeutischer Methoden bei Kindern für die gesamte Verhaltenstherapie ist es um so erstaunlicher, dass 80 Jahre nach diesen Publikationen der Wissensstand zur Wirksamkeit und der Einsatz von Verhaltenstherapie bei Kindern und Jugendlichen immer noch deutlich hinter dem bei Erwachsenen zurückliegt. Die Gründe für die Vernachlässigung psychischer Störungen bei Kindern und Jugendlichen sind vielfältig und können hier nicht abschließend beurteilt werden. Ein bedeutsamer Faktor war aber sicherlich, dass lange Zeit die Sichtweise vorherrschte, viele der Verhaltensprobleme im Kindesalters würden «sich von alleine auswachsen». Dies hat jedoch die moderne Forschung zum Verlauf psychischer Störungen bei Kindern und Jugendlichen nicht bestätigt. So muss man zumindest bei einem Teil der betroffenen Kinder und Jugendlichen von einer Chronifizierung psychischer Störungen bis in das Erwachsenenalter ausgehen. Dieser Befund macht deutlich, dass die Entwicklung psychotherapeutischer Methoden spezifisch für das Kindes- und Jugendalter unbedingt notwendig ist.

Ein weiterer Grund für den Nachholbedarf der Psychotherapieforschung bei psychischen Störungen des Kindes- und Jugendalters ist sicherlich auch, dass diese Forschung aufwendiger und mit mehr ethischen Problemen behaftet ist als die bei Erwachsenen. Es ist daher nachvollziehbar, dass wir für das Kindesalter zwar eine Vielfalt von Behandlungsmethoden zur Verfügung haben, deren Wirksamkeitsnachweise jedoch häufig nicht über Einzelfall-analytische Effektnachweise oder unkontrollierte Gruppenstudien hinausgehen. Nach wir vor stel- len methodisch sorgfältig kontrollierte Gruppenvergleiche noch die Ausnahme und nicht die Regel dar.

In den USA hat das National Institute of Mental Health (NIMH) auf diese alarmierende Situation mit einem Plan zur Verbesserung der Bedingungen für die Erforschung psychischer Störungen des Kindes- und Jugendalters reagiert [s. Members of the Commitee for the Study of Mental Disorders, 1995]. Dieser Plan sieht vor, neben der Verbesserung des Kenntnisstandes zur Epidemiologie, Ätiologie und Therapie psychischer Störungen im Kindes- und Jugendalter die Verbreitung empirisch validierter Behandlungsansätze voranzutreiben. Auch in Deutschland ist die Verbreitung verhaltenstherapeutischer Maßnahmen, die als empirisch validierte Behandlungen gelten, noch völlig unzureichend. Nur die wenigsten Psychotherapeuten, die mit Kindern und Jugendlichen arbeiten, haben eine spezielle Ausbildung für Verhaltenstherapie bei Kindern absolviert. Seit Inkrafttreten des Psychotherapeutengesetzes zum 1. Januar 1999 wächst jedoch die Zahl neuer Weiterbildungsinstitute für Verhaltenstherapie mit Kindern und Jugendlichen. Es ist zu hoffen, dass sich dadurch die Versorgungslage für Kinder und Jugendliche mit psychischen Störungen in Deutschland erheblich verbessern wird.

Das vorliegende Heft möchte zur Verbreitung verhaltenstherapeutischer Methoden im Kindes- und Jugendalter beitragen, indem es einen Überblick über den aktuellen Forschungsstand der Therapie ausgewählter psychischer Störungen in dieser Altersgruppe gibt. Dabei soll der gesamten Bandbreite verhaltenstherapeutischen Vorgehens Rechnung getragen werden: von den stark behavioral orientierten Interventionen, die bereits im Säuglings- und Kleinkindalter zur Anwendung kommen, bis zu den kombinierten kognitiv-verhaltenstherapeutischen Behandlungsansätzen, die eher bei Störungen im Kindes- und Jugendalter eingesetzt werden.

Im ersten Artikel geben Borg-Laufs und Merod eine allgemeine störungsübergreifende Einführung in die speziellen 
Aspekte der Verhaltenstherapie mit Kindern und Jugendlichen. Daran schließen sich fünf Arbeiten an, die das störungsspezifische verhaltenstherapeutische Vorgehen bei den folgenden Störungsbildern beschreiben: Fütterungsprobleme bei Säuglingen und Kleinkindern (Wolke), Hyperkinetische Störungen und Störungen des Sozialverhaltens (Döpfner), Trennungsangst und Phobien (Schneider) und Anorexia nervosa (Steinhausen). Im letzten Beitrag beschreiben Moll und Rothenberger das verhaltenstherapeutische Vorgehen bei Zwangsstörungen und verknüpfen dabei die verhaltenstherapeutischen Maßnahmen mit den Erkenntnissen der neurobiologischen Grundlagenforschung. Dem interessierten Leser soll dieses Heft eine Einführung in die verhaltenstherapeutische
Vorgehensweise und deren Verankerung in der empirischen klinisch-psychologischen sowie kinder- und jugendpsychiatrischen Forschung geben.

\section{S. Schneider, Basel}

\section{Literatur}

Jones M: A laboratory study of fear: The case of Peter. Pedagog Semin J Genetic Psychol 1924:31:308-315.

Members of the Committee for the Study of Research on Child and Adolescent Mental Disorders: Report card on the National Plan for Research on Child and Adolescent Mental Disorders. Arch Gen Psychiatry 1995;52:715-734.

Mowrer OH, Mowrer WM: Enuresis: A method for its study and treatment. Am J Orthopsychiatry 1938;8:436-459. 